\title{
Quark propagator from an improved staggered action in Laplacian and Landau gauges
}

\author{
Patrick O. Bowman ${ }^{\mathrm{a} *}$, Urs M. Heller ${ }^{\mathrm{a}}$ and Anthony G. Williams ${ }^{\mathrm{b}}$ \\ ${ }^{a}$ Department of Physics and CSIT, Florida State University, Tallahassee FL 32306-4120, USA \\ ${ }^{\mathrm{b}} \mathrm{CSSM}$ and Department of Physics and Mathematical Physics, Adelaide University, Australia 5005
}

Studies of gauge dependent quantities are afflicted with Gribov copies, but Laplacian gauge fixing provides one possible solution to this problem. We present results for the lattice quark propagator in both Landau and Laplacian gauges using standard and improved staggered quark actions. The standard Kogut-Susskind action has errors of $\mathcal{O}\left(a^{2}\right)$ while the improved "Asqtad" action has $\mathcal{O}\left(a^{4}\right), \mathcal{O}\left(a^{2} g^{2}\right)$ errors and this improvement is seen in the quark propagator. We demonstrate the application of tree-level corrections to these actions and see that Landau and Laplacian gauges produce very similar results. In addition, we test an ansatz for the quark mass function, with promising results. In the chiral limit, the infrared quark mass, $M\left(q^{2}=0\right)$ is found to be $260 \pm 20$ $\mathrm{MeV}$.

\section{The quark propagator}

The quark propagator is a fundamental quantity of QCD. Though gauge dependent, it manifestly displays dynamical chiral symmetry breaking, contains the chiral condensate and $\Lambda_{\mathrm{QCD}}$, and has been used to compute the running quark mass [1,2]. Some model hadron calculations rely on ansätze for the quark propagator, yet on the lattice we have the opportunity to study it in a direct, nonperturbative fashion. Quark propagator studies can be complicated, however, by strong lattice artefacts [3.4]; it has also been studied using the overlap action [5.

We are required to fix a gauge and we choose the Landau and the Laplacian gauges [6]. We use Wilson glue at $\beta=5.85(a \simeq 0.125 \mathrm{fm})$ on a $16^{3} \times$ 32 lattice and six quark masses from $a m=0.075$ down to 0.0125 (115 to $19 \mathrm{MeV})$. Calculations were done on 80 configurations.

We use the standard Kogut-Susskind (KS) action and the "Asqtad" quark action [9], a fat-link staggered action that combines three-link, fivelink and seven-link staples to minimise flavour changing interactions along with the three-link Naik term and planar five-link Lepage term. The coefficients are tadpole improved and chosen to

\footnotetext{
*Presented by POB.
}

remove all tree-level $\mathcal{O}\left(a^{2}\right)$ errors. This action was motivated by the desire to improve flavour symmetry, but has also been reported to have good rotational properties and small mass renormalisation [10].

In the (Euclidean) continuum, Lorentz invariance allows us to decompose the full quark propagator into Dirac vector and scalar pieces

$$
S^{-1}\left(p^{2}\right)=Z^{-1}\left(p^{2}\right)\left[i \gamma \cdot p+M\left(p^{2}\right)\right] .
$$

Asymptotic freedom means that, as $p^{2} \rightarrow \infty$, $S^{-1}\left(p^{2}\right) \rightarrow i \gamma \cdot p+m$, (the free propagator) where $m$ is the bare quark mass.

From consideration of the tree-level forms of our two lattice actions, we define the momentum variables $q_{\mu} \equiv \sin \left(p_{\mu}\right)$ for the KS action and

$$
q_{\mu} \equiv \sin \left(p_{\mu}\right)\left[1+\frac{1}{6} \sin ^{2}\left(p_{\mu}\right)\right]
$$

for the Asqtad action, where $p_{\mu}$ is the usual lattice momentum,

$$
p_{\mu}=\frac{2 \pi n_{\mu}}{a L_{\mu}} \quad n_{\mu} \in\left[\frac{-L_{\mu}}{4}, \frac{L_{\mu}}{4}\right) .
$$

By considering the propagator as a function of $q_{\mu}$ instead of $p_{\mu}$, we ensure that the lattice quark propagator has the correct tree-level form and 
hopefully better approximates its continuum behaviour. This is the same philosphy that has been used in studies of the gluon propagator (see Ref. 111 and references therein). See also footnote 6 in Ref. [2].

To help us identify lattice artefacts - as we are employing only one set of configurations - we separate the data according to the direction in which the momentum lies. Data from momenta lying wholly on a spatial cartesian direction are plotted with squares, along the temporal direction, triangles, and along the four-diagonal, with diamonds. Circles represent any other combination.

\section{Laplacian gauge}

Laplacian gauge is a nonlinear gauge fixing that respects rotational invariance yet is free of Gribov ambiguities. Although it is difficult to understand perturbatively, it is equivalent to Landau gauge in the asymptotic region [7, 8. It is also computationally cheaper then Landau gauge. There is, however, more than one way of obtaining such a gauge fixing in $S U(N)$. The three implementations of Laplacian gauge fixing used here are:

1. $\partial^{2}(\mathrm{I})$ gauge (QR decomposition), used in Ref. [12].

2. $\partial^{2}(\mathrm{II})$ gauge, where the complex $3 \times 3$ matrix is projected onto $S U(3)$ by maximising its trace. This will be discussed in more detail in an upcoming paper on the gluon propagator [13].

3. $\partial^{2}$ (III) gauge (Polar decomposition), the original prescription described in Ref. [6].

The gauge transformations employed in Laplacian gauge fixing are constructed from the lowest eigenvectors of the covariant lattice Laplacian operator. The way that these eigenvectors transform under gauge transformations provides the uniqueness of the Laplacian gauge. The three implementations discussed differ in the way that the gauge transformation is constructed from those eigenvectors. We can think of each projection method as defining its own Laplacian gauge. In all cases the resulting gauge is unambiguous for all configurations except a set of measure zero.
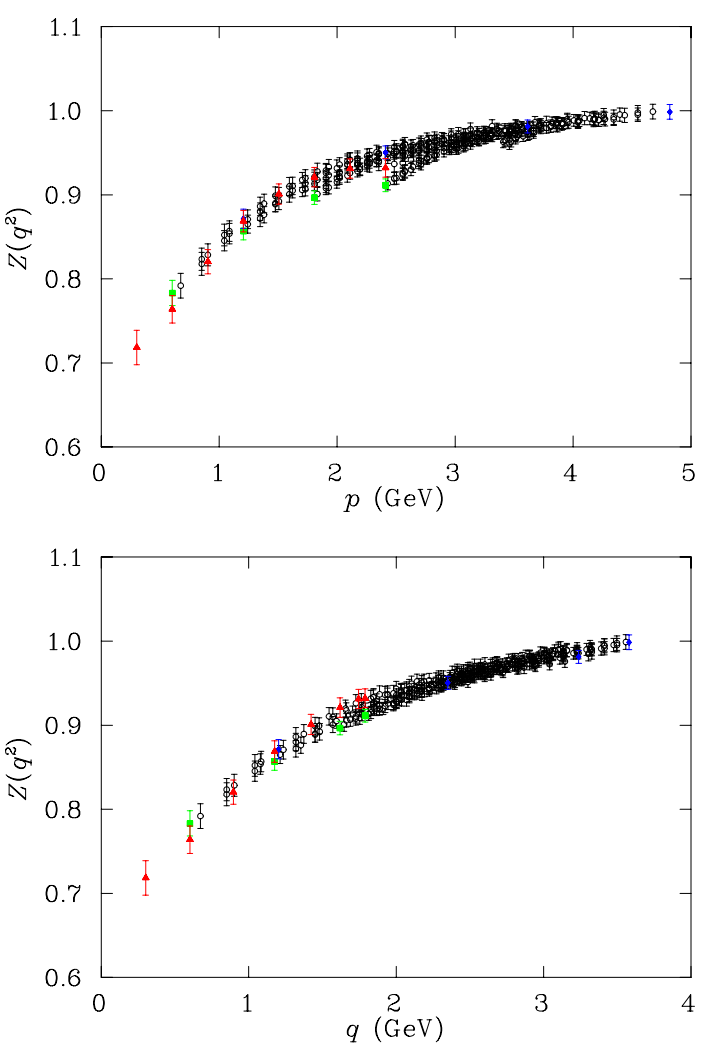

Figure 1. The quark Z function for the Asqtad action $(m a=0.05)$. When we use the momentum $q$, defined by the tree-level form of the action, rotational symmetry breaking is reduced.

\section{Results}

\subsection{Performance of the Asqtad action}

We investigated the applicaton of tree-level correction to the quark propagator by comparing the $Z$ functions using $p$ and $q$. One example is shown in Fig. 1. With the Asqtad action we see that hypercubic artefacts are small in any case, but when we use $q$ instead of $p$ they nearly vanish.

It is less clear which momentum variable should be used for the mass function, because at treelevel it is not multiplied by the momentum, but for consistency we use $q$ here as well. In the case of the mass function, the choice of momentum will actually make little difference to our results.

In Fig. 2 the mass function is plotted, in Landau gauge, for both actions with quark mass 

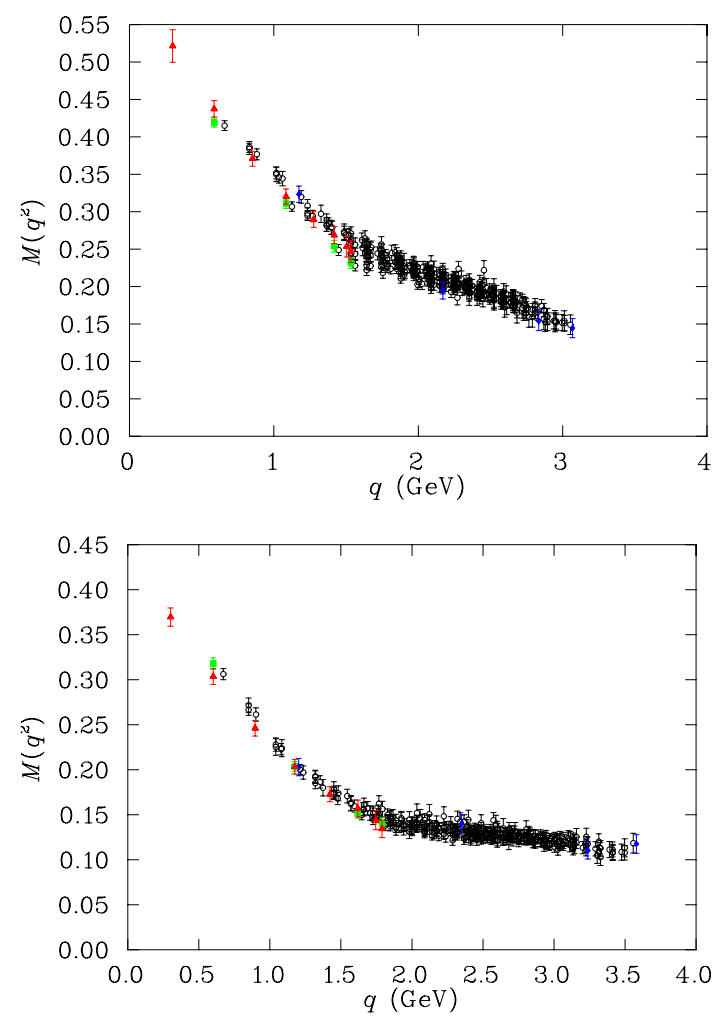

Figure 2. Mass function for quark mass $m a=$ $0.05(m \simeq 77 \mathrm{MeV}), \mathrm{KS}$ action (top) and Asqtad action (bottom) in Landau gauge.

$m a=0.05$. We see that the KS action gives a much larger value for $\mathrm{M}(0)$ than the Asqtad action and is slower to approach asymptotic behaviour. The Asqtad action also shows slightly better rotational symmetry.

The Asqtad action displays clearly better rotational symmetry in the quark $Z$ function and, curiously, improved infrared behaviour as well. The Asqtad action also displays a better approach to asymptopia, more smoothly approaching one in the ultraviolet. Furthermore, the relative improvement increases as the quark mass decreases. Comparing the mass function for the two actions at $m a=0.0125$, the lowest mass studied here, the propagator has much less infrared noise with the Asqtad action than with the KS action.
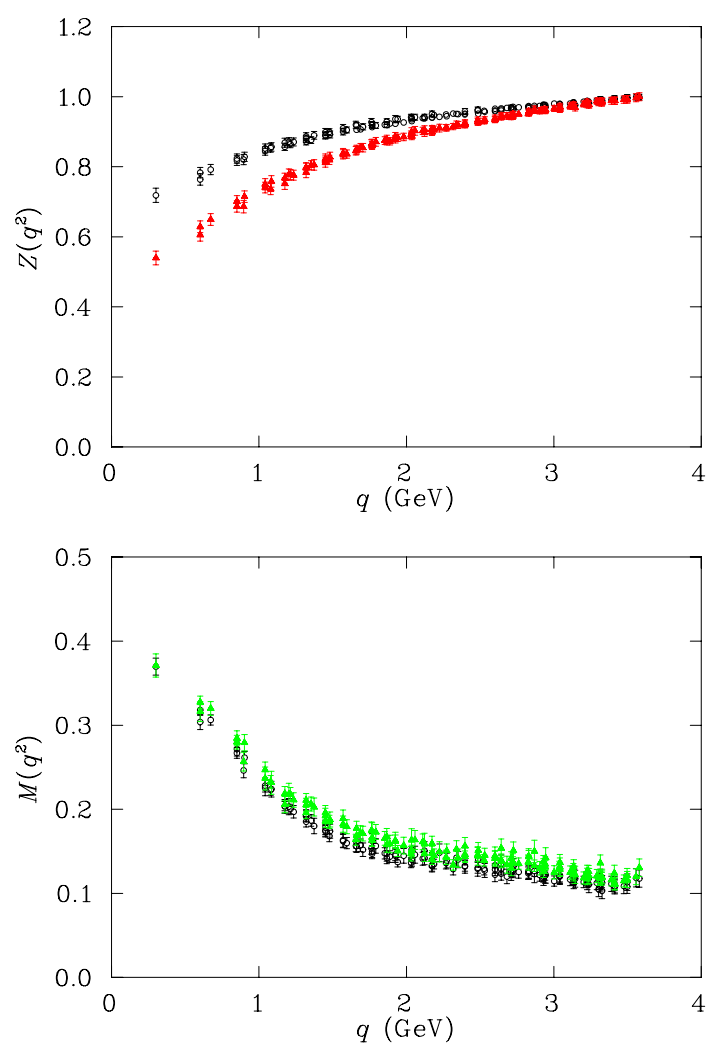

Figure 3. Gauge dependence of the quark Z (top) and mass (bottom) functions for the Asqtad action $(m a=0.05)$. Points marked with open circles are in Landau gauge and solid triangles are in $\partial^{2}(\mathrm{I})$ gauge. Data has been cylinder cut.

\subsection{Comparison of the gauges}

Fig. 3 (top) shows the $Z$ function for the Asqtad action in Landau and $\partial^{2}(\mathrm{I})$ gauges. Data has been cylinder cut 11] for easier comparison. They are in excellent agreement in the ultraviolet but differ significantly in the infrared. The dipping of $Z$ in the infrared is associated with dynamical chiral symmetry breaking. There appears to be some slight difference in the $Z$ function between $\partial^{2}(\mathrm{I})$ and $\partial^{2}(\mathrm{II})$ gauges.

Fig. 3 (bottom) shows the mass function for the Asqtad action in Landau and $\partial^{2}(\mathrm{I})$ gauges. The two mass functions agree in the ultraviolet and in the infrared, but the Landau gauge mass function sits slightly higher in the intermediate region. The mass functions are nearly identical in 


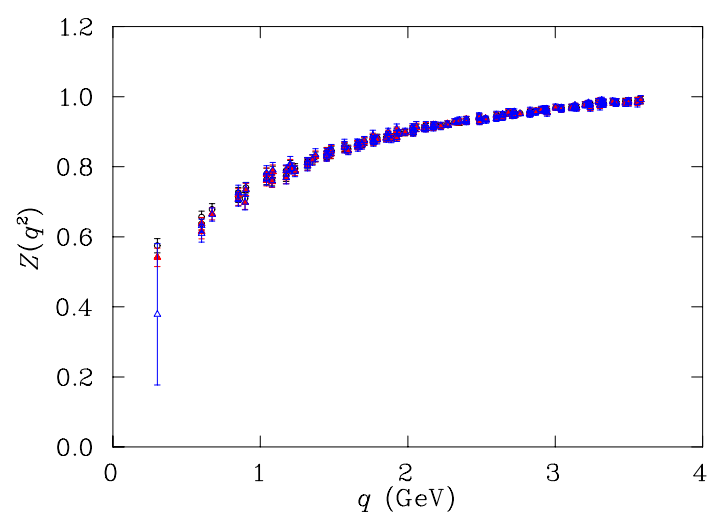

Figure 4. Comparison of the quark $\mathrm{Z}$ functions for the three quark masses $m a=0.0125,0.025$ and 0.05 , with the Asqtad action in $\partial^{2}(\mathrm{II})$ gauge. Data has been cylinder cut. As in Landau gauge, they agree to within errors, although there is a systematic ordering of the infrared points from heaviest quark (top) to lightest (bottom).

$\partial^{2}(\mathrm{I})$ and $\partial^{2}(\mathrm{II})$ gauges. We have also found that in Landau gauge the mass function has slightly less anisotropy at this lattice spacing.

Landau gauge seems to respond somewhat better than $\partial^{2}(\mathrm{II})$ gauge to vanishing quark mass. With the smallest quark mass $(m a=0.0125)$ the lowest momentum points have large errors in $\partial^{2}(\mathrm{II})$ gauge compared with Landau gauge. This can be seen in the $Z$ function in Fig. 4 .

$\partial^{2}(\mathrm{III})$ performs very poorly. We found that many of the matrices had vanishingly small determinants (compared to numerical precision), which destroyed the projection onto $S U(3)$. Problems with $\partial^{2}$ (III) have also been seen in the gluon propagator 13.

\subsection{Modelling the quark propagator}

We performed an extrapolation to the chiral limit using a quadratic fit. The model ansatz

$$
M(q)=\frac{c \Lambda^{1+2 \alpha}}{q^{2 \alpha}+\Lambda^{2 \alpha}}+m_{0},
$$

which is a generalisation of the one used in Ref. [3], was then fit to each mass function. The resulting fit parameters are shown in Table 1 and Fig. 5 shows the best fit for the mass function in the chiral limit. We show here only results for

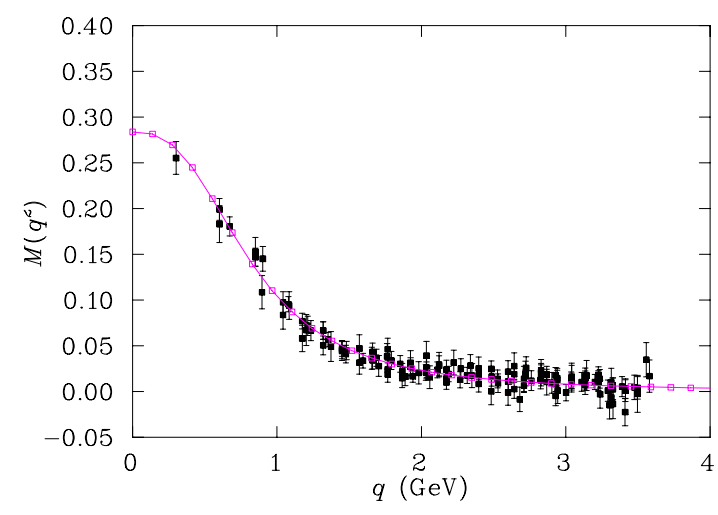

Figure 5. Mass function extrapolated to the chiral limit. Errors are Jack-knife. Fit parameters are $\mathrm{c}=0.030(4), \Lambda=870(60) \mathrm{MeV}, m_{0}=0.0, \alpha=$ $1.52(23), \chi^{2} /$ dof $=0.49$.

Landau gauge, as they are compatible with the Laplacian gauge results, but slightly cleaner. We find that $\alpha>1$ is increasingly favoured as the quark mass approaches zero.

\section{Conclusions}

We have seen that the lattice quark propagator has better rotational symmetry and displays more rapid approach to asymptotic behaviour with the Asqtad action than with the standard Kogut-Susskind action. Three implementations of the Laplacian gauge were investigated, and it was found that $\partial^{2}(\mathrm{I})$ and $\partial^{2}(\mathrm{II})$ gauges gave similar results to Landau gauge. $\partial^{2}(\mathrm{III})$ worked very poorly. The mass function showed very little sensitivity to the choice of gauge, but some change was seen in the quark $\mathrm{Z}$ function. We attempted to model the mass function and saw that the ansatz provided a good fit to the data.

As we have simulated on only one lattice, it remains to do a thorough examination of discretisation and finite volume effects. A natural extension of the mass function ansatz would be to include the correct asymptotic behaviour, and this would require testing on much finer lattices.

\section{Acknowledgments}

The authors wish to thank Derek Leinweber and Jonivar Skullerud for useful discussions. 
Table 1

Best-fit parameters for the ansatz, Eq. (4), in Landau gauge, in physical units. The table is divided into two sections, the first has $\alpha=1.0$ fixed and the second leaves $\alpha$ as a free parameter. For the last fit in each case, the ultraviolet mass, $m_{0}$, was fixed to zero. Generally, $\alpha$ is not well determined by the fits, but $\alpha>1$ seems to be favoured in the chiral limit.

\begin{tabular}{ccccccc}
\hline$m(\mathrm{MeV})$ & $\mathrm{c}$ & $\Lambda(\mathrm{MeV})$ & $m_{0}(\mathrm{MeV})$ & $\alpha$ & $\mathrm{M}(0)(\mathrm{MeV})$ & $\chi^{2} / \mathrm{dof}$ \\
\hline 115 & $0.40(2)$ & $920(20)$ & $144(7)$ & 1.0 & $467(9)$ & 0.38 \\
96 & $0.36(5)$ & $890(70)$ & $118(8)$ & 1.0 & $440(20)$ & 0.42 \\
77 & $0.41(5)$ & $830(70)$ & $95(7)$ & 1.0 & $430(20)$ & 0.42 \\
58 & $0.45(4)$ & $770(50)$ & $70(7)$ & 1.0 & $420(20)$ & 0.51 \\
38 & $0.49(6)$ & $720(60)$ & $44(6)$ & 1.0 & $400(30)$ & 0.56 \\
19 & $0.54(7)$ & $670(60)$ & $18(6)$ & 1.0 & $380(30)$ & 0.69 \\
0 & $0.56(8)$ & $660(50)$ & $-12(6)$ & 1.0 & $350(30)$ & 0.66 \\
0 & $0.77(15)$ & $530(50)$ & 0.0 & 1.0 & $410(40)$ & 1.3 \\
\hline 115 & $0.28(1)$ & $1000(30)$ & $157(7)$ & $1.25(4)$ & $433(7)$ & 0.38 \\
96 & $0.28(2)$ & $975(40)$ & $131(9)$ & $1.26(10)$ & $408(9)$ & 0.37 \\
77 & $0.30(5)$ & $940(50)$ & $110(10)$ & $1.29(6)$ & $380(10)$ & 0.36 \\
58 & $0.30(2)$ & $920(40)$ & $80(6)$ & $1.30(2)$ & $360(10)$ & 0.41 \\
38 & $0.36(6)$ & $830(50)$ & $55(7)$ & $1.28(7)$ & $350(20)$ & 0.46 \\
19 & $0.34(4)$ & $830(100)$ & $28(5)$ & $1.35(14)$ & $310(40)$ & 0.55 \\
0 & $0.30(4)$ & $870(60)$ & 0.0 & $1.52(23)$ & $260(20)$ & 0.49 \\
\hline
\end{tabular}

POB and UMH would like to thank the organisers of the Lattice Hadron Physics workshop (Cairns, 2001) for a great workshop and POB also thanks them for financial support.

\section{REFERENCES}

1. S. Aoki et al., Phys. Rev. Lett. 82, 4392 (1999).

2. D. Becirevic et al., Phys. Rev. D 61, 114507 (2000).

3. J-I. Skullerud and A.G. Williams, Phys. Rev. D 63, 054508 (2001).

4. J. Skullerud et al., Phys. Rev. D 64, 074508 (2001).

5. F.D.R. Bonnet et al., These proceedings.

6. J.C. Vink and U-J. Wiese, Phys. Lett. B 289, 122 (1992).

7. P. van Baal, Nucl. Phys. B (Proc. Suppl.) 42, 843 (1995).

8. J.E. Mandula, hep-lat/0111019.

9. K. Orginos, D. Toussaint and R.L. Sugar, Phys. Rev. D 60, 054503 (1999).

10. J. Hein et al., hep-lat/0110045.

11. F.D.R. Bonnet et al., Phys. Rev. D 64,
034501 (2001).

12. C. Alexandrou, Ph. de Forcrand and E. Follana, Phys. Rev. D 63, 094504 (2001).

13. P.O. Bowman, U.M. Heller, D.B. Leinweber and A.G. Williams, Work in progress. 